\title{
Highly Dynamic Ad hoc on Demand Distance Vector (HDAODV) Routing Protocol for MANET
}

\author{
K.Vanaja \\ Assistant Professor in M.C.A \\ Vivekanandha College of Arts and \\ Sciences for Women, \\ Tiruchengode, Tamil Nadu
}

\author{
Dr. R. Umarani \\ Associate Professor in CS \\ Sri Sarada College for \\ Women, Salem, Tamil Nadu
}

\author{
V.Selvi \\ AssistantProfessor in CS \\ Nehru Memorial College, \\ Puthanampatti,Trichy, \\ Tamil Nadu
}

\begin{abstract}
Mobile ad hoc network is an emerging trend and it has to face many challenges and issues. Routing in these networks is highly complex due to moving nodes and hence many protocols have been developed. Routing in ad hoc networks is a very challenging issue due to nodes mobility, dynamic topology, frequent link breakage, limitation of nodes (memory, battery, bandwidth, and processing power), and lack of central point like base stations or servers. So by analyzing different ad hoc routing protocols based on the metric throughput, packet delivery ratio, end to end delay which may yield a solution to the challenges in the ad hoc routing in different situations. The performance may vary depending upon the topology, flow patterns, mobility speed of the system.

Topology of mobile ad hoc network is varying from time to time. The basic idea behind this paper is to modify AODV that each node uses routing information provided by the new neighbor nodes to find out and exchanges to better paths and create accumulated routes for later use. Simulated modeling results in Network Simulator(NS2) show that the approach can create more optimal routes and significantly improves the performance using by decreasing average delay time in some cases. This paper highlights the swapping of path information on AODV which is highly dynamic (HDAODV) when there is no link breakage. The performance results put HDAODV is bit better than AODV.
\end{abstract}

General Terms

Ad-Hoc, DSDV, AODV, DSR, NS2, Mobile Ad hoc Network (MANET).

\section{Keywords}

Node mobility, dynamic topology, frequent link breakage, throughput, packet delivery ratio, end to end delay.

\section{INTRODUCTION}

Recently, there has been tremendous growth in the sales of laptop and portable computers. These smaller computers nevertheless can be equipped with hundreds of megabytes of disk storage, high resolution color displays pointing devices and wireless communications adapters. Moreover, since many of these small (in size only) computers operate for hours with battery power users are free to move about at their convenience with out being constrained by wires.

As people begin to have mobile computers handy, for whatever purposes, sharing information between the computers will become a natural requirement. Currently, such sharing is made difficult by the need for users to perform administrative tasks and set up static, bidirectional links between their computers. Thus one of our primary motivations is to allow the construction of temporary networks with no wires and no administrative intervention required. In this paper, such a interconnection between the mobile computers will be called an ad hoc network in conformance with current usage within the IEEE 802.11 subcommittee.

Routing protocols for existing networks have not been designed specifically to provide the kind of dynamic self starting behavior needed for ad-hoc networks. Most protocols exhibit their least desirable behavior when presented with a highly dynamic interconnection topology. Although we thought that mobile computers could naturally be modeled as routers, it was also clear that existing routing protocols would place too heavy a computational burden on each mobile computer. Lastly, the wireless medium differs in important ways from wired media, which would require that we make modifications to whichever routing protocol we might choose to experiment with. Since we had to make lots of changes anyway, we decided to follow our ad-hoc network model as far as we could and ended up with a substantially new approach to the classic on demand distance vector routing.

\section{OVERVIEW OF ROUTING METHODS}

In our environment, the problem of routing is essentially the distributed version of the shortest path problem. Each node in the network maintains for each destination a preferred neighbor. Each data packet contains a destination node identifier in its header. When a node receives a data packet it forwards the packet to the preferred neighbor for its destination. The forwarding process continues until the packet reaches its destination. The manner in which routing tables are constructed, maintained and updated differs from one routing method to another. Popular routing methods, however, attempt to achieve the common objective of routing packets along the optimal path. The next -hop routing methods can be 
categorized into two primary classes: link-state and distancevector.

2.1 Link-State The link-state approach is closer to the centralized version of the shortest path computation method. Each node maintains a view of the network topology with a cost for each link. To keep these views consistent, each node periodically broadcasts the link costs of its outgoing links to all other nodes using a protocol such as flooding. As a node receives this information, it updates its view of the network topology and applies a shortest-path algorithm to choose its next hop for each destination. Some of the link costs in a node's view can be incorrect because of long propagation delays, partitioned network etc. Such inconsistent views of network topologies might lead to formation of routing loops. These loops, however, are short lived, because they disappear in the time it takes a message to traverse the diameter of the network.

\subsection{Distance-Vector}

A distance-vector routing protocol uses the Bellman-Ford algorithm to calculate paths. A distance-vector routing protocol requires that a router informs its neighbors of topology changes periodically and, in some cases, when a change is detected in the topology of a network. Compared to link-state method, it is computationally more efficient, easier to implement and requires much less storage space. However, it is well known that this algorithm can cause the formation of both short-lived and Long-lived loops. Almost all proposed modifications to DBF algorithm, eliminate the looping problem by forcing all nodes in the network to participate in some form of inter nodal coordination protocol. Such inter nodal coordination mechanisms might be effective when topological changes are rare.

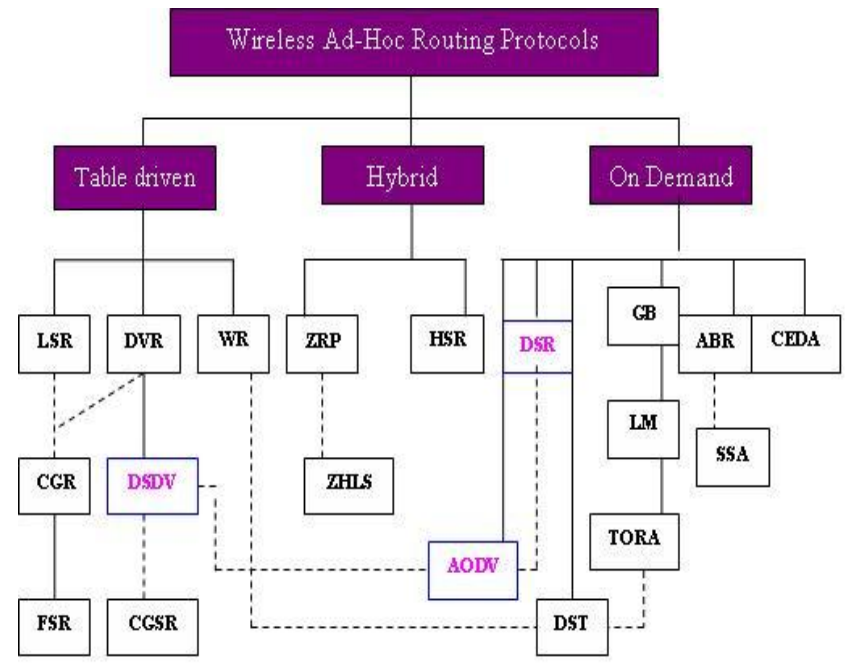

Figure 1 : Classification of Routing protocols

\section{AD HOC ON DEMAND DISTANCE VECTOR (AODV)}

AODV is a relative of the Bellman-Ford distance vector algorithm, but is adapted to work in a mobile environment. $A O D V$ combines the use of destination sequence number
(DSDV) and the on-demand route discovery (DSR). AODV determines a route to a destination only when a node wants to send a packet to that destination. Routes are maintained as long as they are needed by the source. Sequence numbers ensure the freshness of routes and guarantee the loop-free routing.

Our proposed routing method allows a collection of mobile computers, which may not be close to any base station and can exchange data along changing and arbitrary paths of interconnection ,to afford all computers among their number a (possibly multi-hop) path along which data can be exchanged. In addition, our solution must remain compatible with operation in cases where a base station is available. By the methods outlined in this paper, routing will be seen to solve the problems associated with ad-hoc networks nodes on when mobility.

This protocol uses three different messages to discover and maintain links.

\section{- $\quad$ Route Request (RREQ) \\ - Route Reply (RREP) \\ - Route Error (RERR)}

When a route to a new destination is needed, the node broadcasts a RREQ to find a route to the destination. A route can be determined when the RREQ reaches either the destination itself, or an intermediate node with a 'fresh enough' route to the destination. A 'fresh enough' route is a valid route entry for the destination whose associated sequence number is at least as great as that contained in the RREQ. The route is made available by unicasting a RREP back to the origination of the RREQ. Each node receiving the request caches a route back to the originator of the request, so that the RREP can be unicast from the destination along a path to that originator, or likewise from any intermediate node that is able to satisfy the request.

Nodes monitor the link status of next hops in active routes. When a link break in an active route is detected, a RERR message is used to notify other nodes that the loss of that link has occurred. The RERR message indicates those destinations (possibly subnets) which are no longer reachable by way of the broken link.

During route maintenance phase, the nodes that are part of an active route may offer connectivity information by broadcasting local Hello Messages periodically (Special ROUTE REPLY message) to its immediate neighbors. If there is no message arriving from neighbor beyond some threshold time, the connection is assumed to be lost.

When a node detects that a route to a neighbor node is not valid it removes the routing entry and sends a ROUTE ERROR message to neighbors that are active and use the route. This is possible by maintaining active neighbors lists. This procedure is repeated at nodes that receive ROUTE ERROR messages. A source that receives a ROUTE ERROR can reinitiate a ROUTE REQUEST message. 
4. HIGHLY DYNAMIC AODV (HDAODV) The proposed modification of the protocol is due to change in topology by the migration (mobility) of a node which is a part of the path already.

Nodes in AODV do not update the current link if it is not broken. It may slack many optimal paths. Each node in AODV maintains a routing table and neighbor list [1]. Knowing one node's routing table and neighbor list may lead to discover a new optimal route (with less number of hops) or accumulated paths [3] through that node. Accumulated paths are not ondemand paths but can be discovered with no additional cost. There is possibility that the next on-demand destination can be reached by one of those accumulated paths. In this case, source can transmit packet immediately instead of discovering a new route. This can reduce the delay time and routing overhead for the network.
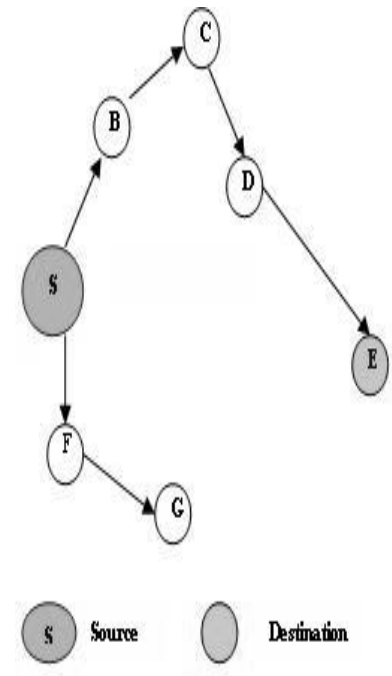

Path From S to $\mathrm{E}: \mathrm{S} \rightarrow \mathrm{B} \rightarrow \mathrm{C} \rightarrow \mathrm{D} \rightarrow \mathrm{E}$

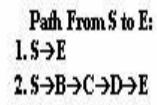

Figure 2: Proposed Scenario

This paper proposed a modification by incorporating the learning information from new neighbor nodes mechanism in AODV which is termed as Highly dynamic AODV (HDAODV). In order to evaluate the new modification, we created detail packet levels simulations in NS2 to compare its performance with the original one. AODV can be modified to use effectively the routing information provided by the new neighbor nodes. Each time a node discovered a new neighbor node. These two nodes exchange the necessary information. For each routing table entry, we extracted destination address, number of hop towards that destination, sequence number and expire time of that entry. The extracted entries are formed into a destination table to exchange with the new neighbor node.

For each entry, look up its destination address in the routing table. If the destination is found, it means that besides the current path in the routing table, there has an alternate path through the new neighbor node. The number of hops of these two paths is then compared. Consider the number of hops of the old path and new path are hopold and hopnew respectively.
If hopold $>$ hop new then

alternate path is better (with smaller number of hops),

swap the current path by new path;

otherwise

No change

End if

If the destination is not found in the routing table and neighbor list, an entry toward that destination is created in the routing table as a new accumulated The update and accumulated path both obtain the sequence number, number of hop and expire time from the destination table.

With this modification, source can discover and change to a better path even if the current path is not broken. The accumulated paths will also decrease the number of Route Discovery cycles and reduce the delay time for finding a path. This design therefore improves the performance of AODV.

\section{SIMULATIONS AND RESULTS}

The simulations use 5 different movement patterns (pause time $0,20,40,100$ seconds) and 4 different traffic patterns $(5,10$, 15 , and 20 sources). These patterns create 20 scenarios; each scenario combines a movement pattern and a traffic pattern. A wide variety of node scenario files and CBR scenario files were generated to evaluate varying network conditions. The results are as follows based on average time delay as metric.

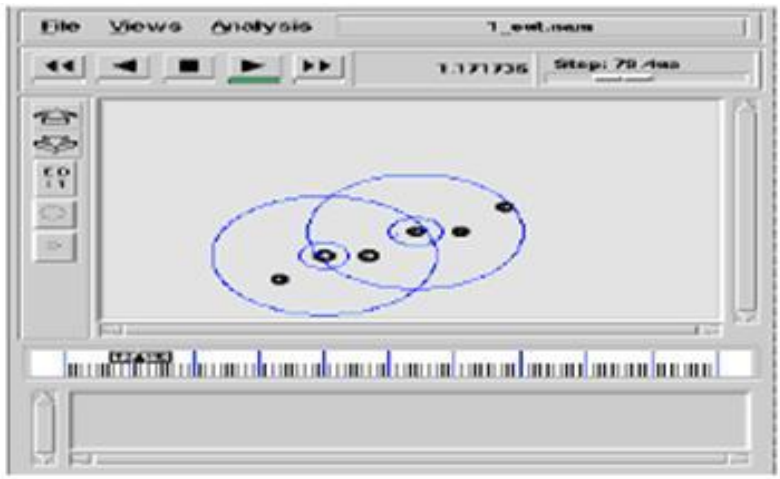

Figure 3: NAM Screen with Ad Hoc Network

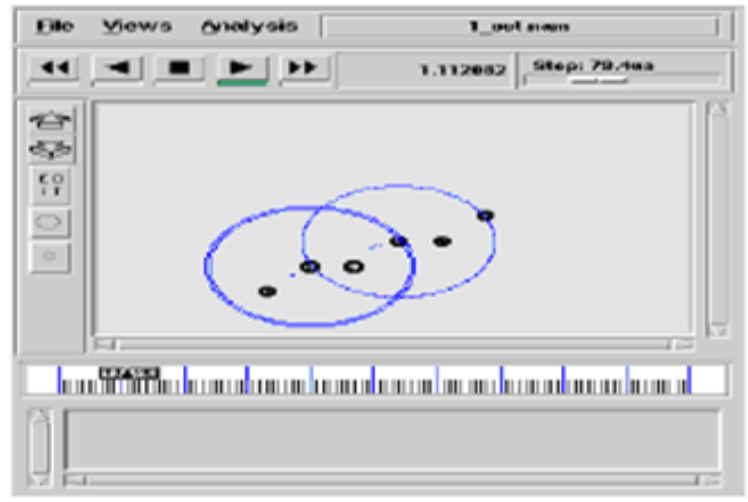

Figure 4: NAM Screen with Packet transfer 


\subsection{Average Time Delay}

The below figure 5 shows the comparison of average time delay of AODV and HDAODV. The modified solution has less time delay in some of the cases. It is because HDAODV creates accumulated paths, therefore, with high traffic the possibility of using accumulated paths increases. Original AODV does not have accumulated paths so it will take time to discover the route if traffic required is not found in the routing table.

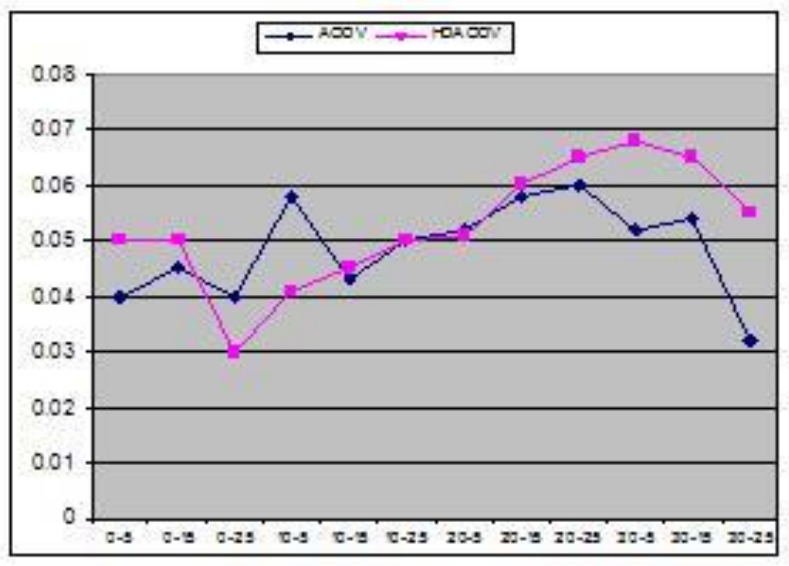

Figure 5: Average Time Delay Vs Traffic Movement

\subsection{Packet Delivery Ratio}

Figure 5 presents the comparison of Packet Delivery Ratio. We can see that HDAODV performs better in most of the case. The reason is HDAODV updates the path immediately when it has chance to do so while original AODV keeps the link until it is broken. Because of that, there will be more broken link in original AODV, which creates more packet loss than the one in modified AODV. Packet Delivery ratio (PDR $)^{[4]}=(($ Number of Packets
Received/Number of Packet Sent $) * 100)$

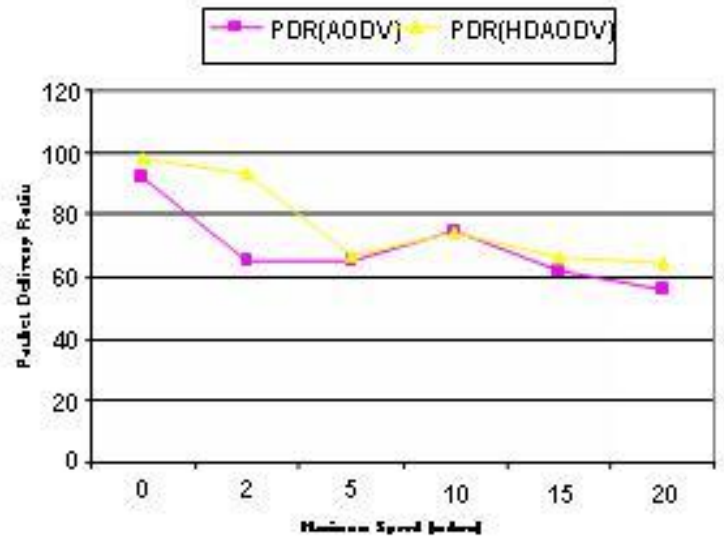

Figure 4: Packet Delivery Ratio : AODV Vs HDAODV

\section{CONCLUSION}

Providing convenient connectivity for mobile computers, in adhoc computers is a challenge that is only now being met. We have presented an innovative approach of AODV, which models the mobile computers as routers, which are cooperating to forward packets as needed to each other. We make good use of the properties of the wireless broadcast medium. Our approach can be utilized for the environment with less scalability and mobility.

In this paper the AODV routing protocol has been reviewed. As a reactive protocol AODV transmits network information only on-demand. Though the current path is not broken, the proposed protocol HDAODV which swaps path information if exists an optimal path.

\section{FUTURE WORK}

The implemented preliminary version of this change in the protocol AODV can be utilized with mobile computers, with less scalability. Currently we are making necessary modifications using NS2 simulator, for use in creating the appropriate simulation environment for our needs. We hope to discover good operational values via simulation using other metrics such as control overhead, routing overhead with different mobility patterns.

\section{ACKNOWLEDGMENTS}

First I thank Almighty for giving me the necessary wisdom to accomplish this research work. I profoundly thank Dr.R.Umarani,, who has suggesting to carry out this work in an innovative way. I extend my grateful thanks to Mr.G.VijayKumar, Serive -IT, iNautix Technologies India Private Limited, Chennai who has supporting on my research and friends who are in the research circle and the peer review committee members of IJCA for their valuable comments.

\section{REFERENCES}

[1] Le Anh Tuan, Yuan Luo " Exchange Routing information between neighbor nodes to improve AODV performance", 2009 Sith International Conference on Information Technology: New generations.-IEEE.

[2] C. Perkin, Elizabeth M. Royer, S. Das, "Ad hoc on demand Distance Vector Routing”, RFC 3561, July 2003, http://www.ietf.org/rfc/rfc3561.txt

[3] S. R. Das, C. E. Perkins, E. M. Royer, and M. K. Marina, "Performance Comparison of Two On- Demand Routing Protocols for Ad Hoc Networks," in IEEE Personal Communications Magazine special issue on Ad hoc Networking, February 2001, pp. 16-22.

[4] S. Gwalani, E. Royer, Charles E. Perkins, "AODVPA: AODV with Path Accumulation", Next Generation Internet Symposium, held in conjunction with ICC, Anchorage, Alaska, May 2003.

[5] E. Royer and C. K. Toh, "A Review of Current Routing Protocols for Ad-Hoc Mobile Wireless Networks", in IEEE Personal Communications, April 1999, pp.46-55.

[6] E. Royer, Charles E. Perkins, "Evolution and future directions of the ad hoc on-demand distance- vector 
routing protocol", Ad Hoc Networks Journal, Vol. 1 No. 1, July 2003, pp. 125-150.

[7] C. S. R. Murthy and B. S. Manoj, Ad Hoc Wireless Networks: Architecture and Protocols, ch. Routing Protocols for Ad Hoc Wireless Networks, pp. 299-364. Prentice Hall Communications Engineering and Emerging Technologies Series, New Jersey: Prentice Hall Professional Technical Reference, 2004.

\section{AUTHORS BIOGRAPHY}

K. Vanaja received her M.Phil., degree from Bharathidasan University, Trichy in July 2005. She is working as Assistant Professor in M.C.A. Department, Vivekanandha College of Arts and Sciences for women, Tiruchengode, Tamil Nadu. India. She is currently guiding M.C.A,

M.Phil research scholars of various universities. She has presented 10 papers in National Conference and 2 papers in International Conference and 3 in journals. Her areas of interest include Networks - information security, mobile communication, and Artificial Intelligence.
Dr. R.Uma Rani received her Ph.D., Degree from Periyar University, Salem in the year 2006. She is a rank holder in M.C.A., from NIT, Trichy. She has published around 40 papers in reputed journals and national and international conferences. She has received the best paper award from VIT, Vellore, Tamil Nadu in an international conference. She has done one MRP funded by UGC. She has acted as resource person in various national and international conferences. She is currently guiding 5 Ph.D., scholars. She has guided 20 M.Phil., scholars and currently guiding 4 M.Phil., Scholars. Here areas of interest include information security, data mining, fuzzy logic and mobile computing.

Mrs V.Selvi received her M.Phil., degree from Bharathidasan University, Trichy in July 2004. She is working as Lecturer in Computer Science Department, Nehru Memorial College, Puthanampatti, Trichy, Tamil Nadu. India. She is currently guiding M.C.A, M.Phil research scholars of various universities. She has presented 10 papers in National Conference and 2 papers in International Conference and 3 in journals. Her areas of interest include Networks - information security, mobile communication, and Data Structure. 